Review

\title{
Endothelial dysfunction contributes to COVID-19-associated vascular inflammation and coagulopathy
}

Jun Zhang ${ }^{1, *}$, Kristen M. Tecson ${ }^{1}$ and Peter A. McCullough ${ }^{1,2,3}$

\author{
${ }^{1}$ Baylor Heart and Vascular Institute, Dallas, TX 75226, USA \\ ${ }^{2}$ Baylor University Medical Center, Dallas, TX 75226, USA \\ ${ }^{3}$ Baylor Jack and Jane Hamilton Heart and Vascular Hospital, Dallas, TX 75226, USA \\ *Correspondence: Zhangi37@gmail.com (Jun Zhang)
}

DOI: $10.31083 /$ i.rcm.2020.03.126

This is an open access article under the CC BY 4.0 license (https://creativecommons.org/licenses/by/4.0/).

Great attention has been paid to endothelial dysfunction (ED) in coronavirus disease 2019 (COVID-19). There is growing evidence to suggest that the angiotensin converting enzyme 2 receptor (ACE2 receptor) is expressed on endothelial cells (ECs) in the lung, heart, kidney, and intestine, particularly in systemic vessels (small and large arteries, veins, venules, and capillaries). Upon viral infection of ECs by severe acute respiratory syndrome coronarvirus 2 (SARS-CoV-2), ECs become activated and dysfunctional. As a result of endothelial activation and ED, the levels of pro-inflammatory cytokines (interleukin -1, interleukin-6 (IL$6)$, and tumor necrosis factor- $\alpha$ ), chemokines (monocyte chemoattractant protein-1), von Willebrand factor (vWF) antigen, vWF activity, and factor VIII are elevated. Higher levels of acute phase reactants (IL-6, C-reactive protein, and D-dimer) are also associated with SARS-CoV-2 infection. Therefore, it is reasonable to assume that ED contributes to COVID-19-associated vascular inflammation, particularly endotheliitis, in the lung, heart, and kidney, as well as COVID-19-associated coagulopathy, particularly pulmonary fibrinous microthrombi in the alveolar capillaries. Here we present an update on ED-relevant vasculopathy in COVID-19. Further research for ED in COVID-19 patients is warranted to understand therapeutic opportunities.

\section{Keywords}

COVID-19; coagulation; cytokines; endothelial dysfunction; SARS-CoV2; von Willebrand factor; thrombosis

\section{Understanding endothelial dysfunction}

Generally, endothelial dysfunctioned (ED) involves functional phenotype modifications in order to regulate hemostasis and thrombosis and control inflammatory reactions within blood vessels (Gimbrone and García-Cardeña, 2016). In regard to an acute pro-inflammatory response, endothelial activation, particularly in small vessels, is divided into two types: type I endothelial activation (stimulation) and type 2 endothelial activation, which involve effector proteins including class-II major histocompatibility antigens, E-selectin, vascular cell adhesion molecule-1 (VCAM-
1), tissue factor, interleukin-8 (IL-8) and monocyte chemotractant protein-1 (MCP-1) (Gimbrone and García-Cardeña, 2016). These proteins are detectable in the microvasculature at sites of inflammation.

From a pathological perspective, ED consists of type 1 and type II endothelial activation, as well as endothelial cell injury (e.g., endothelial cell apoptosis and necrosis), as demonstrated by pathological findings in animal studies (Zhang et al., 2012). The following terms "endothelial activation", "endothelial dysfunction", "endothelial damage" and "endothelial injury" may be used interchangeably (Zhang et al., 2017).

\section{The sequence of cellular events leading to endothelial dysfunction}

The sequence of cellular events leading to ED may be described as (1) reversible type I endothelial activation (endothelial stimulation), which represents an immediate event of ED that release prostored proteins vWF, P-electin, thrombin, histamine, etc; (2) reversible type II endothelial activation (over hours or days), which synthesizes new proteins such as CRP, fibrinogen, vWF, tissue factor, E-selectin, intercellular adhesion molecule-1 (ICAM-1), vascular cell adhesion molecule-1 (VCAM-1), IL-1, and MCP-1; (3) irreversible endothelial apoptosis, which leads to detachment of ECs by anoikis (a form of programmed cell death, as manifested by their detaching from the surrounding extracellular matrix); and (4) irreversible endothelial necrosis. In both endothelial apoptosis and necrosis, increased plasma levels of thrombomudulin and vWF further lead to endothelial damage (Fig. 1) (Zhang et al., 2010). Furthermore, ED may be associated with vascular EC injury in the development of vasculitis and thrombosis.

\section{Biomarkers of endothelial dysfunction}

Previous work outlined a panel of soluble mediators as biomarkers of endothelial activation in drug-induced vascular injury (Zhang et al., 2010). Vascular inflammation is mediated by endothelial adhesion molecules: E-selectin (endothelial-leukocyte adhesion molecule 1), immunoglobulin gene superfamily (ICAM-1, VCAM-1, etc.), pro-inflammatory cytokines (TNF$\alpha$, IL-6), and pro-inflammatory chemokine (IL-8). Conversely, vascular coagulation is mediated by $\mathrm{P}$-selectin and a pro-coagulant 


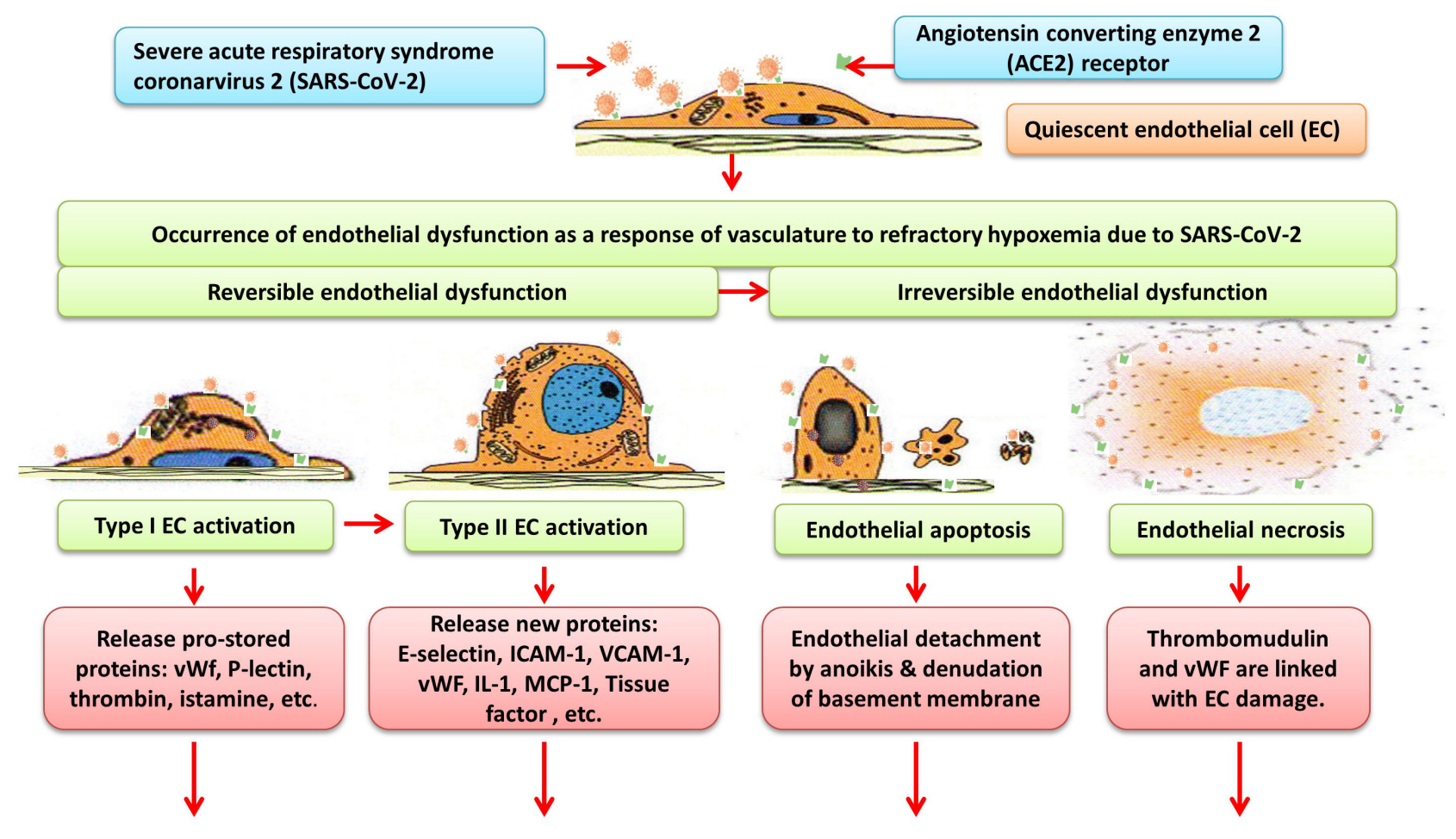

\section{COVID-19 - associated vascular inflammation and COVID-19 - associated coagulopathy}

Fig. 1. Schematic diagram depicting endothelial dysfunction (ED) contributing to COVID-19-associated vascular inflammation and coagulopathy. The angiotensin converting enzyme 2 (ACE2) receptor is widely expressed on endothelial cells in the lung, heart, kidney, and intestine, allowing endothelial cells (EC) to be infected by severe acute respiratory syndrome coronarvirus 2 (SARS-CoV-2). The sequence of cellular events leading to ED begins (as early as) immediately for type I EC activation, followed by type II EC activation, EC apoptosis, and EC necrosis. Type I EC activation does not require de novo protein synthesis by means of immediately releasing pro-stored proteins such as von Willebrand factor (vWF), P-selectin, thrombin, and histamine. Conversely, type II EC activation requires de novo protein synthesis by means of releasing new proteins such as vWF, Tissue factor, fibrinogen, E-selectin, intercellular adhesion molecule-1 (ICAM-1), vascular cell adhesion molecule-1 (VCAM-1), interleukin-1 (IL-1), monocyte chemoattractant protein-1, (MCP-1), and C-reactive protein. Endothelial apoptosis results in endothelial detachment by anoikis \& denudation of basement membrane, whereas endothelial necrosis results in further release of thrombomudulin and vWF. Finally, leukocyte adhesion molecules, pro-inflammatory cytokines (IL-1, IL-6, and TNF), chemokines (MCP-1), together with pro-coagulant molecules contribute to COVID-19 --associated inflammation and coagulopathy

family (vWF, vWF pro-peptite, plasminogen activating inhibitor-1 (PAI-1)). Type I EC activation results in the loss of anticoagulant molecules, compared to type II EC activation, which leads to the de novo synthesis of procoagulant molecules. The anticoagulant (antithrombotic) molecules consist of thrombomodulin, tissue plasminogen activator, heparin sulfate, and antithrombin. The procoagulant (prothrombotic) molecules include thrombin, PAI-1, vWF, tissue factor and platelet activating factor. While anticoagulant and procoagulant activities are balanced in tissue factor, thromboxane A2 (TXA2), the imbalance associated with the loss of anticoagulant molecules and synthesis of procoagulant molecules favors thrombosis (Zhang et al., 2010).

It is worth noting that $\mathrm{P}$-selectin is released from Weibel-Palade bodies of activated ECs' cytoplasm and also from a-granules of activated platelets. The vWF and vWF pro-peptides are also released from Weibel-Palade bodies of activated ECs as well as platelets, megakaryocytes, and plasma. Furthermore, thrombomodulin is released from activated ECs as well as monocytes, macrophages, and leukocytes (Zhang et al., 2010). Therefore, the activities of both pro- and anti-coagulant molecules are involved in activated ECs, platelets, and megakaryocytes. Furthermore, it is suggested that when thrombomodulin is suppressed, IL- $\alpha$, IL- $\beta$, and TNF-a are prothrombotic and hinder what would otherwise be anticoagulant effects from thrombomodulin-dependent activation of protein S and protein C (Zhang et al., 2010).

Indeed, increased levels of pro-inflammatory cytokines (e.g., IL-2R, IL-6, TNF- $\alpha$ ) were reported in patients diagnosed with severe COVID-19 (Chen et al., 2020). Therefore, these cytokines play a central role in inducing the loss of normal antithrombotic and anti-inflammatory functions of ECs. The levels of vWF antigen, factor VIII and P-selectin were dramatically elevated in patients with COVID-19-associated coagulopathy, particularly in the ICU, indicating activation of ECs and platelets. In contrast, the levels of soluble thrombomodulin and endogenous anticoagulants (antithrombin and proteins $\mathrm{C}$ and $\mathrm{S}$ ) were not significantly different between ICU patients and controls (Lyss, 2020). Therefore, the culprit in COVID-19-associated coagulopathy is endothelial activation in association with activation of platelets and megakary- 
ocytes, thereby inducing imbalance between pro-coagulant and anti-coagulant activities.

\section{Endothelial activation and dysfunction in COVID-19}

Escher et al. (2020) reported a 72-year-old previously healthy male patient who presented with acute respiratory distress syndrome. Continual increases of D-dimer (a marker of fibrin degradation) were observed on day 4 , day 11 , and day 21 . On day 21 , the patient had significant elevation of vWF, with vWF: antigen and vWF: activity, as well as increased Factor VIII clotting activity. The profile of increased vWF family likely represents reversible type I endothelial activation (endothelial stimulation) and type II cell activation, as described in the preceding section. The authors therefore suggest that endothelial activation is likely mediated by the ACE2 receptor.

A pathological study of COVID-19 by Varga et al. (2020) identified viral inclusion bodies in a peritubular space and viral particles in ECs of the glomerular capillary loops by using electron microscopy. The characteristic appearance of aggregates of viral particles is that of a dense circular surface and lucid center. In addition, an immunohistochemical staining of caspase 3 revealed apoptosis of ECs and apoptotic bodies in both small intestine and lung sections. Further, post-mortem histology displayed an accumulation of inflammatory cells associated with endothelium in the heart, small bowel, and lymphocytic endotheliitis in the lung, heart, kidney, liver, and small intestine. The study illustrates a prominent feature of ED occurring in COVID-19. First, ECs can directly be infected by SARS-CoV-2 because the ACE2 receptor is expressed widely in ECs. Second, after direct viral infection of ECs, ED leads to diffuse vascular inflammation (endotheliitis). Third, over time, reversible ED (endothelial activation) progresses to irreversible ED (endothelial apoptosis and necrosis). Ultimately, ED induces vascular dysfunction of different organs.

Ackermann et al. (2020) utilized immunohistochemical staining to discover a significantly larger average number of ACE2positive ECs per field of view $(0.55 \pm 0.11)$ in capillaries from lungs of patients with COVID-19, compared with those from patients with influenza and healthy controls $(0.49 \pm 0.28$ and 0.066 \pm 0.03 , respectively). Electron microscopy detected SARS-CoV2 within the cell membrane of ECs. Histopathologic examination revealed a series of endothelial lesions, which are consistent with the morphology of endothelial activation, dysfunction, and injury (Ackermann et al., 2020). Pathological findings of cell swelling, severe endothelial injury, disruption of intercellular junctions, and basal membrane contact loss in COVID-19 patients imply that the destruction of ECs leads to pulmonary vascular endotheliitis and alveolar capillary microthrombi (Ackermann et al., 2020).

\section{COVID-19-associated vascular inflammation and coagulopathy}

COVID-19-induced endotheliitis may be attributed to endothelial adhesion molecules (selectins, ICAM-1, VCAM-1), proinflammatory cytokines (IL-6, IL-2 receptor, and TNF- $\alpha$ ), and proinflammatory chemokines (MCP-1), which can be released by activated and dysfunctional ECs (Fig. 1). These mediators could participate in leukocyte recruitment in the microvasculature. In a systemic review of ED, Pons et al. (2020) emphasized that in severe SARS-CoV-2 infections, ECs plays a crucial role of ED in vascular dysfunction, immunothrombosis and inflammation.

There is evidence to support increased levels of ED-released mediators not only induce ED, but also induce a prothrombotic state. It is noteworthy that micro- and macrovascular thrombosis both are present in venous and arterial circulations, and that venous thrombotic events (e.g., pulmonary embolism) are associated with elevated D-dimer in COVID-19 patients. Klok et al. (2020) reported a $31 \%$ incidence of thrombotic complications for patients in the intensive care unit with COVID-19 infections. Tang et al. (2020) reported abnormal coagulation parameters, including significantly higher levels of D-dimer and fibrin degradation product, longer prothrombin time and activated partial thromboplastin time in the non-survivors with COVID-19. Léonard-Lorant et al. (2020) demonstrated that patients with pulmonary embolus had higher Ddimer levels than those without. Upon autopsy, Fox et al. (2020) discovered that thrombotic microangiopathy in the small vessels and capillaries of the lungs contributed to death in patients with severe COVID-19. As in others' findings, D-dimer levels were markedly elevated (Fox et al., 2020). Pulmonary microthrombi were characterized by the appearance of small and firm thrombus, multi foci and within small pulmonary artery and venule of the cut section of peripheral parenchyma (Fox et al., 2020). Thrombosed small vessels were immunostained with vWF; however, CD61 immunostains also showed additional fibrin, platelet thrombus, and positive stained megakaryocytes (Fox et al., 2020). These findings suggest that pulmonary thrombosis is involved in other cellular events, platelets, and megakaryocytes. The mechanisms for pulmonary thrombosis are likely manifold in COVID-19 patients; however, it is presumed that ED is an initial major event, which can further trigger activation of platelets and megakaryocytes, thereby causing an abnormal anticoagulant status. Ackermann et al. (2020) discovered widespread thrombosis with microangiopathy, which is characterized by multiple fibrinous microthrombi, in the alveolar capillaries and postcapillary venules. Finally, a review by Becker (2020) provides a timely examination of COVID-19associated coagulopathy, highlighting distinctions from disseminated intravascular coagulopathy and thrombotic microangiopathy and reiterates that COVID-19-associated coagulopathy is marked by elevated D-dimer levels in the peripheral blood, indicating a breakdown of fibrin polymers by plasmin.

\section{Increasing evidence of COVID-19-associated vascular inflammation and coagulopathy}

There have been autopsy findings to suggest that COVID-19 is associated with vascular inflammation and coagulopathy. Pulmonary pathology from 23 autopsies of COVID-19 decedents was characterized by acute interstitial pneumonia with diffuse alveolar damage (Buja et al., 2020). Another notable feature in that examination was fibrin-rich thrombi within capillaries and small blood vessels, as well as large pulmonary thromboemboli (Buja et al., 2020). Further, thrombotic microangiopathy and pulmonary thromboembolism in the lungs, along with endotheliitis and microvascular dysfunction in the cardiovascular system, and virus induced pro-coagulant state and coagulopathy and predisposition 
of deep vein thrombosis and pulmonary thromboembolism in the hematologic system were observed (Buja et al., 2020). In another autopsy report in the United States, Magro et al. (2020) noted a pattern of tissue damage consistent with complement-mediated microvascular injury in the lung and/or skin of 5 individuals with severe COVID-19. Their results highlight the role of complement activation and microvascular thrombosis in cases of persistent, severe COVID-19. Microthrombi, attributed to ED, within small lung arteries and prostate were reported in Germany (Wichmann et al., 2020). Glomerular capillary microthrombi were observed in both kidneys from a patient with COVID-19 in Japan, indicating early signs of disseminated intravascular coagulation (Adachi et al., 2020). In addition, fibrinous thrombi in alveolar arterioles were reported at autopsy in Brazil (Nunes Duarte-Neto et al., 2020). The authors suspected that virus-induced EC dysfunction may have been involved, but it was not determined at the time of publication (Nunes Duarte-Neto et al., 2020). An examination in Italy considered post-mortem wedge liver biopsies from 48 patients who died from severe pulmonary COVID-19 and respiratory failure. The authors found partial or complete luminal thrombosis of portal and sinusoidal vessels, indicating that not only the lungs, but also the endothelial layer of blood vessels were targeted by the virus (Sonzogni et al., 2020).

In non-autopsy studies, coagulopathy is a common abnormality in patients with COVID-19. For example, a case of acute portal vein thrombosis, which is accompanied by a pro-thrombotic profile of increased levels of vWF, D-dimer, and fibrinogen in addition to increased levels of IL-6 and C-reactive protein, was reported in Italy. That case was characterized by sepsis-related hypotension and portal vein thrombosis relevant to impairment of arterial and venous hepatic blood flow; the patient's symptoms rapidly improved following anticoagulation (La Mura et al., 2020). In France, 69\% of anticoagulated patients with severe COVID-19 illness had venous thromboembolism (Llitjos et al., 2020).

\section{Endothelial cells contribute to innate and adaptive immunity in COVID-19}

ECs may serve as antigen-presenting cells. By expressing major histocompatibility complex (MHC) class I and MHC II antigens, ECs contribute to innate and adaptive immune responses. However, the number of T cells activated by ECs is far fewer than those activated by professional antigen-presenting cells. Cytokine-induced endothelial activation and cell adhesion in the endothelium, as well as cytokine-increased MHC class I or II molecules in the endothelium have been studied in this context. Cytokines, such as the interferon family, TNF family, IL-1, and IL-2 increase MHC class I or II antigen expression in the endothelium. For example, TNF can enhance IFN- $\gamma$-induced MHC class I and II antigen expression, inducing CD8 T cell activation (Virzì et al., 2018).

ED and endothelial activation likely co-determine an uncontrolled immune response in COVID-19 (Teuwen et al., 2020). However, many questions remain to be determined. Among these questions are (1) to what extent this subtype of capillary ECs is involved in the immune response against SARS- CoV-2 infection (Teuwen et al., 2020) (2) how ECs act synergistically with other antigen-presenting cells to actively participate in innate and adap- tive immune responses; and (3) how much ECs stimulate proliferation of CD4 and CD 8 lymphocytes in COVID-19. Further investigation is needed to prove the effect of ECs in immune response.

\section{Conclusions}

Evidence is emerging to suggest that ED is a contributing factor to vasculopathy and coagulopathy in COVID-19. The vascular endothelium and the various manifestations of its dysfunction in the context of COVID-19 have been considered to be cardinal features. Further studies regarding the role of ED in COVID-19 may have important clinical implications for possible therapeutic interventions.

\section{Authors' contributions}

JZ designed and drafted this work. KMT and PMC critically revised this work. All authors gave final approval.

\section{Acknowledgments}

This work was partially funded by the Baylor Health Care System Foundation.

\section{Conflict of interest}

The authors declare no conflicts of interest statement.

Submitted: July 02,2020

Revised: August 10, 2020

Accepted: August 12, 2020

Published: September 30, 2020

\section{References}

Ackermann, M., Verleden, S. E., Kuehnel, M., Haverich, A., Welte, T., Laenger, F., Vanstapel, A., Werlein, C., Stark, H., Tzankov, A., Li, W. W., Li, V. W., Mentzer, S. J. and Jonigk, D. (2020) Pulmonary Vascular endothelialitis, thrombosis, and angiogenesis in Covid-19. New England Journal of Medicine 383, 120-128.

Adachi, T., Chong, J., Nakajima, N., Sano, M., Yamazaki, J., Miyamoto, I., Nishioka, H., Akita, H., Sato, Y., Kataoka, M., Katano, H., Tobiume, M., Sekizuka, T., Itokawa, K., Kuroda, M. and Suzuki, T. (2020) Clinicopathologic and immunohistochemical findings from autopsy of patient with COVID-19, Japan. Emerging Infectious Diseases 26, 2157 2161

Becker, R. C. (2020) COVID-19 update: Covid-19-associated coagulopathy. Journal of Thrombosis and Thrombolysis 50, 54-67.

Buja, L. M., Wolf, D. A., Zhao, B., Akkanti, B., McDonald, M., Lelenwa, L., Reilly, N., Ottaviani, G., Elghetany, M. T., Trujillo, D. O., Aisenberg, G. M., Madjid, M. and Kar, B. (2020) The emerging spectrum of cardiopulmonary pathology of the coronavirus disease 2019 (COVID19): Report of 3 autopsies from Houston, Texas, and review of autopsy findings from other United States cities. Cardiovascular Pathology $\mathbf{4 8}$ 107233

Chen, G., Wu, D., Guo, W., Cao, Y., Huang, D., Wang, H., Wang, T., Zhang, X., Chen, H., Yu, H., Zhang, X., Zhang, M., Wu, S., Song, J., Chen, T., Han, M., Li, S., Luo, X., Zhao, J. and Ning, Q. (2020) Clinical and immunological features of severe and moderate coronavirus disease 2019. Journal of Clinical Investigation 130, 2620-2629.

Escher, R., Breakey, N. and Lämmle, B. (2020) Severe COVID-19 infection associated with endothelial activation. Thrombosis Research 190 62.

Fox, S. E., Akmatbekov, A., Harbert, J. L., Li, G., Quincy Brown, J. and Vander Heide, R. S. (2020) Pulmonary and cardiac pathology in African American patients with COVID-19: an autopsy series from New Orleans. The Lancet Respiratory Medicine 8, 681-686.

Gimbrone, M. A. and García-Cardeña, G. (2016) Endothelial cell dysfunc- 
tion and the pathobiology of atherosclerosis. Circulation Research $\mathbf{1 1 8}$, 620-636.

Klok, F. A., Kruip, M. J. H. A., van der Meer, N. J. M., Arbous, M. S., Gommers, D. A. M. P. J., Kant, K. M., Kaptein, F. H. J., van Paassen, J., Stals, M. A. M., Huisman, M. V. and Endeman, H. (2020) Incidence of thrombotic complications in critically ill ICU patients with COVID19. Thrombosis Research 191, 145-147.

La Mura, V., Artoni, A., Martinelli, I., Rossio, R., Gualtierotti, R., Ghigliazza, G., Fusco, S., Ierardi, A. M., Andrisani, M. C., Carrafiello, G. and Peyvandi, F. (2020) Acute portal vein thrombosis in SARS-CoV-2 infection. American Journal of Gastroenterology (inpress).

Léonard-Lorant, I., Delabranche, X., Séverac, F., Helms, J., Pauzet, C., Collange, O., Schneider, F., Labani, A., Bilbault, P., Molière, S., Leyendecker, P., Roy, C. and Ohana, M. (2020) Acute pulmonary embolism in patients with COVID-19 at CT angiography and relationship to D-dimer levels. Radiology 296, E189-E191.

Llitjos, J., Leclerc, M., Chochois, C., Monsallier, J., Ramakers, M., Auvray, M. and Merouani, K. (2020) High incidence of venous thromboembolic events in anticoagulated severe COVID-19 patients. Journal of Thrombosis and Haemostasis 18, 1743-1746.

Lyss, A. D. (2020) Endothelial injury may play a major role in COVID19-associated-coagulopthy. Medscape. Available at: https://www. medscape.com/viewarticle/933141 (Accessed: June 29, 2020)

Magro, C., Mulvey, J. J., Berlin, D., Nuovo, G., Salvatore, S., Harp, J., Baxter-Stoltzfus, A. and Laurence, J. (2020) Complement associated microvascular injury and thrombosis in the pathogenesis of severe COVID-19 infection: A report of five cases. Translational Research 220, 1-13.

Nunes Duarte-Neto, A., de Almeida Monteiro, R. A., da Silva, L., Malheiros, D., de Oliveira, E. P., Theodoro Filho, J., Pinho, J., Soares Gomes-Gouvêa, M., Salles, A., de Oliveira, I., Mauad, T., do Nascimento Saldiva, P. H. and Dolhnikoff, M. (2020) Pulmonary and systemic involvement of COVID-19 assessed by ultrasound-guided minimally invasive autopsy. Histopathology (in press).

Pons, S., Fodil, S., Azoulay, E. and Zafrani, L. (2020) The vascular endothelium: the cornerstone of organ dysfunction in severe SARS-CoV2 infection. Critical Care 24, 353.
Sonzogni, A., Previtali, G., Seghezzi, M., Grazia Alessio, M., Gianatti, A. Licini, L., Morotti, D., Zerbi, P., Department of Biomedical and Clinical Sciences University of Milan Milano Italy, Carsana, L., Rossi, R. Lauri, E., Pellegrinelli, A., Nebuloni, M. and Department of Biomedical and Clinical Sciences University of Milan Milano Italy (2020) Liver histopathology in severe COVID 19 respiratory failure is suggestive of vascular alterations. Liver International 40, 2110-2116.

Tang, N., Li, D., Wang, X. and Sun, Z. (2020) Abnormal coagulation parameters are associated with poor prognosis in patients with novel coronavirus pneumonia. Journal of Thrombosis and Haemostasis 18, 844-847.

Teuwen, L., Geldhof, V., Pasut, A. and Carmeliet, P. (2020) COVID-19: the vasculature unleashed. Nature Reviews Immunology 20, 389-391.

Varga, Z., Flammer, A. J., Steiger, P., Haberecker, M., Andermatt, R., Zinkernagel, A. S., Mehra, M. R., Schuepbach, R. A., Ruschitzka, F. and Moch, H. (2020) Endothelial cell infection and endotheliitis in COVID-19. the Lancet 395, 1417-1418.

Virzì, G., Zhang, J., Nalesso, F., Ronco, C. and McCullough, P. (2018) The role of dendritic and endothelial cells in cardiorenal syndrome. Cardiorenal Medicine 8, 92-104.

Wichmann, D., Sperhake, J., Lütgehetmann, M., Steurer, S., Edler, C., Heinemann, A., Heinrich, F., Mushumba, H., Kniep, I., Schröder, A. S., Burdelski, C., de Heer, G., Nierhaus, A., Frings, D., Pfefferle, S., Becker, H., Bredereke-Wiedling, H., de Weerth, A., Paschen, H., Sheikhzadeh-Eggers, S., Stang, A., Schmiedel, S., Bokemeyer, C., Addo, M. M., Aepfelbacher, M., Püschel, K. and Kluge, S. (2020) Autopsy findings and venous thromboembolism in patients with COVID19. Annals of Internal Medicine 173, 268-277.

Zhang, J., Bottiglieri, T. and McCullough, P. A. (2017) The central role of endothelial dysfunction in cardiorenal syndrome. Cardiorenal Medicine 7, 104-117.

Zhang, J., DeFelice, A. F., Hanig, J. P. and Colatsky, T. (2010) Biomarkers of endothelial cell activation serve as potential surrogate markers for drug-induced vascular injury. Toxicologic Pathology 38, 856-871.

Zhang, J., Hanig, J. P. and De Felice, A. F. (2012) Biomarkers of endothelial cell activation: Candidate markers for drug-induced vasculitis in patients or drug-induced vascular injury in animals. Vascular Pharmacology 56, 14-25. 